\title{
Resveratrol ameliorates cardiac dysfunction induced by pressure overload in rats via structural protection and modulation of $\mathrm{Ca}^{2+}$ cycling proteins
}

Qi Dong ${ }^{1,2+}$, Zhiye $\mathrm{Wu}^{2,3+}$, Xiaoyun $\mathrm{Li}^{4}$, Jianyun Yan ${ }^{3}$, Luning Zhao ${ }^{5}$, Chuntao Yang ${ }^{1}$, Junjiang Lu ${ }^{6}$, Ju Deng ${ }^{2,4}$ and Minsheng Chen ${ }^{2,3^{*}}$

\begin{abstract}
Background: Cardiac hypertrophy is a compensatory stage of the heart in response to stress such as pressure overload (PO), which can develop into heart failure (HF) if left untreated. Resveratrol has been reported to prevent the development of hypertrophy and contractile dysfunction induced by PO. However, other studies found that resveratrol treatment for a longer period of time failed to regress cardiac hypertrophy. The aim of this study is to determine the timing of resveratrol treatment to achieve antihypertrophic effect and investigate whether resveratrol prevents the development of HF through preservation of myocardium structure and modulation of $\mathrm{Ca}^{2+}$ handling proteins.
\end{abstract}

Methods: To generate rats with cardiac hypertrophy, male Sprague-Dawley rats were subjected to PO (aortic banding procedure) for 4 weeks. Sham-operated animals served as controls. Rats with cardiac hypertrophy were given resveratrol (4 mg/kg/day) for 4, 6, and 8 weeks, respectively. Histological and echocardiographic analysis and transmission electron microscopy were performed to assess cardiac structure and function. The levels of $\mathrm{Ca}^{2+}$ handling proteins were measured by western blot analysis.

Results: Histological analysis showed that resveratrol treatment regressed developed cardiac hypertrophy at 8 and 10 weeks postsurgery, but not at 12 weeks. However, resveratrol strongly and continuously prevented the development of cardiac dysfunction and dilation of cardiac chamber as evaluated by echocardiography and H\&E staining of heart cross-sections. In addition, PO-induced cardiac fibrosis was completely inhibited by resveratrol treatment. Resveratrol markedly prevented the disrupted myocardium but partially rescued mitochondrial abnormality in banded rats. Moreover, resveratrol prevented the alteration of $\mathrm{Ca}^{2+}$ handling proteins induced by aortic banding, including downregulation of sarcoplasmic reticulum $\mathrm{Ca}^{2+} \mathrm{ATPase}_{2}\left(\mathrm{SERCA}_{2}\right)$ and ryanodine receptor $2\left(\mathrm{Ry}_{2}\right)$, hypophosphorylated phospholamban (PLB), upregulation of $\mathrm{Na}^{+} / \mathrm{Ca}^{2+}$-exchangers $\left(\mathrm{NCX}_{1}\right)$ and increased expression and phosphorylation of $\mathrm{Ca}^{2+} / \mathrm{calmodulin}$-dependent protein kinase II (CaMKII). Moreover, resveratrol alleviated the decreased SERCA activity induced by aortic banding.

Conclusions: Resveratrol effectively prevented the transition from compensatory to decompensatory stage of cardiac hypertrophy induced by $\mathrm{PO}$, but this effect is dependent on the timing of treatment. We suggest that resveratrol may exert beneficial effects on cardiac hypertrophy through protection of cardiac structure and modulation of $\mathrm{Ca}^{2+}$ handling proteins.

Keywords: Resveratrol, Heart failure, Hypertrophy, Ultrastructure, $\mathrm{Ca}^{2+}$ handling proteins

\footnotetext{
*Correspondence: min_sheng_chen@hotmail.com

${ }^{\dagger}$ Equal contributors

${ }^{2}$ Guangzhou Institute of Cardiovascular Disease, Guangzhou 510260, China

${ }^{3}$ Southern Medical University, 1838 Guang zhou da dao bei, Guangzhou

510515, China

Full list of author information is available at the end of the article
}



(c) 2014 Dong et al.; licensee BioMed Central Ltd. This is an Open Access article distributed under the terms of the Creative Commons Attribution License (http://creativecommons.org/licenses/by/4.0), which permits unrestricted use, distribution, and reproduction in any medium, provided the original work is properly credited. The Creative Commons Public Domain Dedication waiver (http://creativecommons.org/publicdomain/zero/1.0/) applies to the data made available in this article, unless otherwise stated. 


\section{Background}

Heart failure (HF) is a major cause of hospitalization and mortality worldwide [1]. Increased hemodynamic load is one of the major contributors to the development of HF. Cardiac hypertrophy is considered to be an adaptive response to pressure or volume overload, which is characterized by the enlargement of heart muscle. Although the initial phase is beneficial in maintaining cardiac function, prolonged hypertrophy will lead to deleterious consequences and eventually HF. Nowadays, many medicines, such as $\beta$-adrenergic receptor blockers, angiotensin-converting enzyme inhibitors, angiotensinreceptor blockers and diuretics, have been universally used to treat cardiac hypertrophy and HF. These medicines can be effective to partially relieve the symptoms of HF, but cannot reverse the progression of HF. In fact, mortality rates of HF still approach about $20 \%$ per year [1]. Therefore, it is critically important to explore alternative approaches to prevent or reverse the progression of cardiac hypertrophy and HF.

Previous studies including The Dietary Approaches to Stop Hypertension (DASH) trial and Lyon Diet Heart Study have demonstrated that increased intake of fruits and vegetables lowered blood pressure in patients with hypertension, reduced the incidence of cardiovascular diseases, and improved survival after myocardial infarction [2]. Furthermore, several studies reported that resveratrol (trans-3, 5, 49- trihydroxystilbene), which is a phenolic phytoalexin and presents in grapes, berries and red wine, alleviated contractile dysfunction, reversed pressure overload (PO)-induced cardiac hypertrophy $[1,3,4]$ and reduced cardiovascular mortality [5]. Wojciechowski P [1] and Juric D [2] found that resveratrol treatment for 2 weeks regressed cardiac hypertrophy in aortic banded rats. However, Ste'phanie Rimbaud demonstrated that resveratrol treatment for 8 weeks did not counteract cardiac hypertrophy in the Dahl salt-sensitive (DSS) rats fed with a high-salt diet (HS-NT) [5]. These inconsistent results indicated that resveratrol may reverse cardiac hypertrophy in a timing-dependant manner. In this study, we investigated the optimal timing of resveratrol treatment to achieve antihypertrophic effect in rats subjected to PO.

Additionally, it is well demonstrated that resveratrol protects against the development of HF by improving contractile functions [1-5]. However, precise mechanisms of its action remain unclear. The normal myocardial structure is essential for cardiac contraction. The most prominent ultrastructural alteration in HF is the loss of contractile elements, which significantly results in reduced cardiac function. Therefore, it is worthwhile to investigate whether resveratrol ameliorates ultrastructural abnormalities in failing hearts induced by PO.

$\mathrm{Ca}^{2+}$ cycling, a critical process referring to the mobilization of intracellular $\mathrm{Ca}^{2+}$ in excitation-contraction (EC) coupling, determines cardiac contractility and relaxation. It is now generally accepted that defective $\mathrm{Ca}^{2+}$ handling proteins in the cycling play an important role in HF pathophysiology. The key defects in $\mathrm{Ca}^{2+}$ cycling occur at the level of the sarcoplasmic reticulum (SR), a $\mathrm{Ca}^{2+}$ storage bulk in muscle. The $\mathrm{SR} \mathrm{Ca}^{2+}$ release channel (ryanodine receptor ${ }_{2}, \mathrm{RyR}_{2}$ ) is oxidized, nitrosylated, and hyperphosphorylated, resulting in "leaky" channels and depletion of $\mathrm{Ca}^{2+}$ from the SR in failing hearts. Downregulated SR $\mathrm{Ca}^{2+}$ ATPase $_{2 \mathrm{a}}\left(\mathrm{SERCA}_{2 \mathrm{a}}\right)$ and hypophosphorylated phospholamban (PLB) contribute to impaired SR $\mathrm{Ca}^{2+}$ uptake that conspires with leaky $\mathrm{RyR}_{2}$ to deplete $\mathrm{SR} \mathrm{Ca}^{2+}$ in failing hearts [6]. Accordingly, the other goal of this study is to verify the hypothesis that resveratrol fixes $\mathrm{Ca}^{2+}$ handling proteins in SR to preserve contractile function in rats subjected to PO.

\section{Methods}

\section{Animal model}

The investigation conforms to the Guide for the Care and Use of Laboratory Animals published by the US National Institutes of Health (NIH Publication No. 8523, revised 1985). Male Sprague-Dawley rats weighing 80-100 g were obtained from Central Animal Care Services at Southern Medical University and subjected to the abdominal aortic banding procedure for induction of PO. Briefly, rats were kept in a room with constant temperature of $26^{\circ} \mathrm{C}$, humidity of $55 \%$, and a $12 \mathrm{~h}$ light: $12 \mathrm{~h}$ dark cycle throughout the study. Animals were given standard rat chow and tap water ad libitum. All rats were anesthetized for surgery with $10 \%$ chloral hydrate $(3 \mathrm{ml} / \mathrm{kg})$. A midline laparotomy was performed, and the suprarenal abdominal aorta was exposed. The aorta between the branches of celiac artery and anterior mesenteric artery was tied off by a $4-0$ silk suture with a blunt 24-gauge needle as a guide. Sham-operated rats were served as controls and subjected to the same surgeries except for the creation of the aortic band. Resveratrol treatment started from 4 weeks postsurgery when LV hypertrophy ocurred, as indicated by the results of echocardiography, and aortic banded rats were divided into treated and untreated subgroups. Resveratrol (Sigma, $4 \mathrm{mg} / \mathrm{kg} / \mathrm{d}$ ) was suspended in $0.5 \%$ carboxymethylcellulose (CMC, Sigma) dissolved in $0.9 \%$ saline. This solution was administered to treated rats by oral gavages for 4, 6 and 8 weeks, respectively. $0.9 \%$ saline was used as a vehicle to treat sham and banded animals.

\section{Echocardiography}

At 4, 8, 10 and 12 weeks postsurgery, rats from all groups were weighed and anesthetized with chloral hydrate. Transthoracic two-dimensionally guided M-mode echocardiography was performed using an IE33 echocardiographic system (Philips Medical Systems, Nederland 
B.V) equipped with a $15-\mathrm{MHz}$ (s12) transducer. The following parameters were measured: percentage of left ventricular (LV) fractional shortening (FS), LV ejection fraction (EF), maximal velocity through left ventricular outflow tract (Vmax), Cardiac output, LV pressure halftime, LV internal dimensions at both diastole and systole (LVIDd and LVIDs, respectively), LV posterior wall dimensions at both diastole and systole (LVPWd and LVPWs, respectively), and interventricular septal dimensions at both diastole and systole (IVSd and IVSs, respectively).

\section{Organ weight and histological analysis}

Body weight, heart and LV with septum were weighed, and the LV mass-to-body weight ratio $(\mathrm{LVm} / \mathrm{BW})$ was calculated. LV samples were fixed in $10 \%$ neutral buffered formalin, embedded with liquid paraffin, and sectioned into $6 \mu \mathrm{m}$ in thickness. Myocyte cross-sectional area was used for the evaluation of the degree of LV hypertrophy. Briefly, sections were stained with hematoxylin and eosin (HE) and examined under a light microscope (Nikon ECLIPSE Ti-U). Five random fields from each of 4 sections per animal were analyzed, and $10 \sim 15$ myocytes per section were measured. The quantification of diameter and area of myocytes were determined with Image Pro Plus 6.0 (Media Cybernetics, Carlsbad, CA). To assess fibrosis, sections were stained with a Masson trichrome kit (Baso, BA4079) according to manufacturer's instructions and stained sections were examined under a light microscope. Interstitial and perivascular fibrosis content were quantified in tissue sections using Image Pro Plus 6.0 as the percentage of connective tissue per crosssectional surface area. The cross sectional area of LV chamber was also measured.

\section{Transmission Electron Microscopy (TEM)}

Hearts were perfused and fixed in $2.5 \%$ glutaraldehyde in $0.1 \mathrm{M}$ sodium cacodylate buffer followed by postfixation with $2 \%$ osmium tetroxide in $0.1 \mathrm{M}$ phosphate buffer ( $\mathrm{pH}$ 7.4) for $2 \mathrm{~h}$ at room temperature. The tissue cubes were then dehydrated in an ascending series of ethanol solutions, followed by acetone dehydration. The tissue cubes were embedded with Embed 812 resin overnight at $37^{\circ} \mathrm{C} .40 \sim 60 \mathrm{~nm}$ ultrathin sections were cut on an ultramicrotome, picked up on grids, and stained with a solution of lead citrate and uranyl acetate. The sections were then observed, and photographs were taken under a Hitachi 7650 electron microscope.

\section{Western blot analysis}

LV tissues frozen in liquid nitrogen were pulverized and homogenized in a lysis buffer containing $20 \mathrm{mM}$ Tris$\mathrm{HCl}\left(\mathrm{pH}\right.$ 7.5), $150 \mathrm{mM} \mathrm{NaCl}, 1 \mathrm{mM} \mathrm{Na}{ }_{2}$ EDTA, $1 \mathrm{mM}$ EGTA, 1\% Triton, $2.5 \mathrm{mM}$ sodium pyrophosphate, $1 \mathrm{mM}$ beta-glycerophosphate, $1 \mathrm{mM} \mathrm{Na} \mathrm{VO}_{4}, 1 \mu \mathrm{g} / \mathrm{ml}$ leupeptin
(20 ml/g tissue) and $1 \mathrm{mM}$ phenylmethylsulfonyl fluoride. Protein samples $(20 \sim 25 \mu \mathrm{g})$ were separated by sodium dodecyl sulfate polyacrylamide gel electrophoresis (SDSPAGE) and transferred to polyvinylidene difluoride (PVDF) membranes. PVDF membranes were probed with the following primary antibodies: anti- $\mathrm{Ca}^{2+} /$ calmodulindependent protein kinase II (CaMKII) antibody (Abcam, 1:1000 dilution), anti-phospho-CaMKII (Thr286) antibody (p-CaMKII, CST, 1:1000 dilution), anti-PLB antibody (Abcam, 1:1000 dilution), anti-phospho-PLB (S16) antibody (p-PLB, Abcam, 1:1500 dilution), anti-SERCA ${ }_{2}$ antibody (Abcam, 1:1000 dilution), anti-RyR $\mathrm{R}_{2}$ antibody (Abcam, 1:1000 dilution) or anti- $\mathrm{Na}^{+} / \mathrm{Ca}^{2+}$-exchangers $\left(\mathrm{NCX}_{1}\right)$ antibody (Abcam, 1:1000 dilution). Appropriate secondary antibodies were used to detect the corresponding primary antibodies, and the antibody-antigen complexes in all membranes were detected by the ECL PLUS kit (Bio-Rad). The protein bands were quantified by Image Pro Plus 6.0.

\section{SERCA activity measurements}

Samples of the left ventricle were prepared as the method previously described by Lizotte E [7]. The ATPase activity in samples was assessed with an ATPase activity assay kit (Nanjing Jiancheng Bioengineering Institute, Nanjing, China) based on a colorimetric estimation of ATP hydrolysis-produced inorganic phosphate. According to manufacturer's instructions, the reactions were initiated by adding samples into the ATPase activity assay buffer. Following $10 \mathrm{~min}$-reaction at $37^{\circ} \mathrm{C}$, the contents of inorganic phosphate in samples were determined by optical densities (OD) measured with the Infinite M1000 PRO plate reader (Tecan, Switzerland) at $636 \mathrm{~nm}$. The protein concentration in samples was determined by BCA kit (Pierce Company). The enzyme activity was defined as the contents of inorganic phosphorus $(\mu \mathrm{mol})$ per mg total protein and hour.

\section{Statistical analysis}

All statistical analyses were performed using the SPSS Statistical software (version 17.0). Values are expressed as mean \pm standard error (SEM). One-way analysis of variance was used to analyze the differences between the means of groups, followed by Tukey post hoc test. A value of $P<0.05$ was considered significant.

\section{Results}

\section{Establishment of hypertrophic model}

Cardiac structure and function in rats were assessed by echocardiography at 4 weeks postsurgery (Additional file 1A). The parameters of LV wall thickness including IVSs, IVSd, LVPWs and LVPWd were significantly increased in aortic banded rats compared with sham rats (Additional file 1B). On the contrary, LVIDs was significantly decreased in aortic banded rats compared with sham rats, whereas no significant difference in LVIDd was 
detected between the two groups (Additional file 1C). Moreover, the parameters of systolic function (EF and FS) were significantly increased in banded rats compared to sham rats (Additional file 1D). These data indicated that the rat model of compensatory cardiac hypertrophy induced by aortic banding was successfully established.

\section{Antihypertrophic effect of resveratrol}

Histological analysis showed that surface areas and diameters of cardiomyocytes were remarkably increased at 8,10 and 12 weeks postsurgery in aortic banded rats compared with sham rats, but decreased by $27 \%, 14 \%$ at 8 weeks and $46 \%, 26 \%$ at 10 weeks postsurgery respectively in resveratrol-treated rats compared to banded rats. However, we found that resveratrol treatment failed to reverse enlargement in cardiomyocytes at 12 weeks postsurgery (Figure $1 \mathrm{~A}-\mathrm{C}$ ).

The $\mathrm{LVm} / \mathrm{BW}$ was increased by $29 \%, 54 \%$ and $59 \%$ in banded rats at 8,10 and 12 weeks postsurgery respectively compared to sham rats. The $\mathrm{LVm} / \mathrm{BW}$ in resveratroltreated rats was not significantly different at 8 weeks postsurgery and significantly increased by $35 \%$ and $26 \%$ at 10 and 12 weeks postsurgery compared with sham rats, whereas was lower than banded rats at 10 and 12 weeks (Figure 1D).

\section{Effect of resveratrol on LV chamber}

H\&E staining analysis of cardiac cross-sections revealed dilation of LV in banded rats at 8, 10 and 12 weeks postsurgery. Resveratrol treatment showed a significant alleviation of LV dilation induced by aortic band at indicated time point (Figure 2A, B). Consistent with the histological data, LVIDd and LVIDs detected by echocardiographic analysis were also increased in banded rats compared with sham rats at 10 and 12 weeks postsurgery, and this effect was eliminated by resveratrol treatment (Figure 2C, D).

\section{Effect of resveratrol on cardiac systolic function}

Echocardiographic analysis of cardiac function was carried out at 8,10 , and 12 weeks postsurgery in sham, banded and resveratrol-treated rats. We found that systolic functional parameters (EF, FS and Vmax) in banded rats were decreased continuously compared to sham rats at 8,10 , and 12 weeks postsurgery. Of note, resveratrol treatment significantly increased EF by $14 \%, 15 \%, 26 \%$, FS by $30 \%, 32 \%, 26 \%$, and Vmax by $30 \%, 26 \%, 25 \%$ at 8 , 10,12 weeks postsurgery respectively, compared with banded rats. FS and Vmax in resveratrol-treated rats were even remarkably higher than sham rats at 12 weeks postsurgery (Figure 3).


Figure 1 Effects of resveratrol on the size of cardiomyocytes $(n=3)$ and the LV mass-to-body weight ratio $(L V m / B W)(n=6)$.

(A) Photomicrographs of left ventricular tissue sections stained by hematoxylin and eosin. (B) Myocyte diameter. (C) Myocyte area. (D) LVm/BW. Data are mean \pm SEM. ${ }^{*} P<0.05^{* *} P<0.01$ vs. sham rats; ${ }^{\dagger} P<0.05{ }^{\dagger \dagger} P<0.01$ vs. banded rats. 

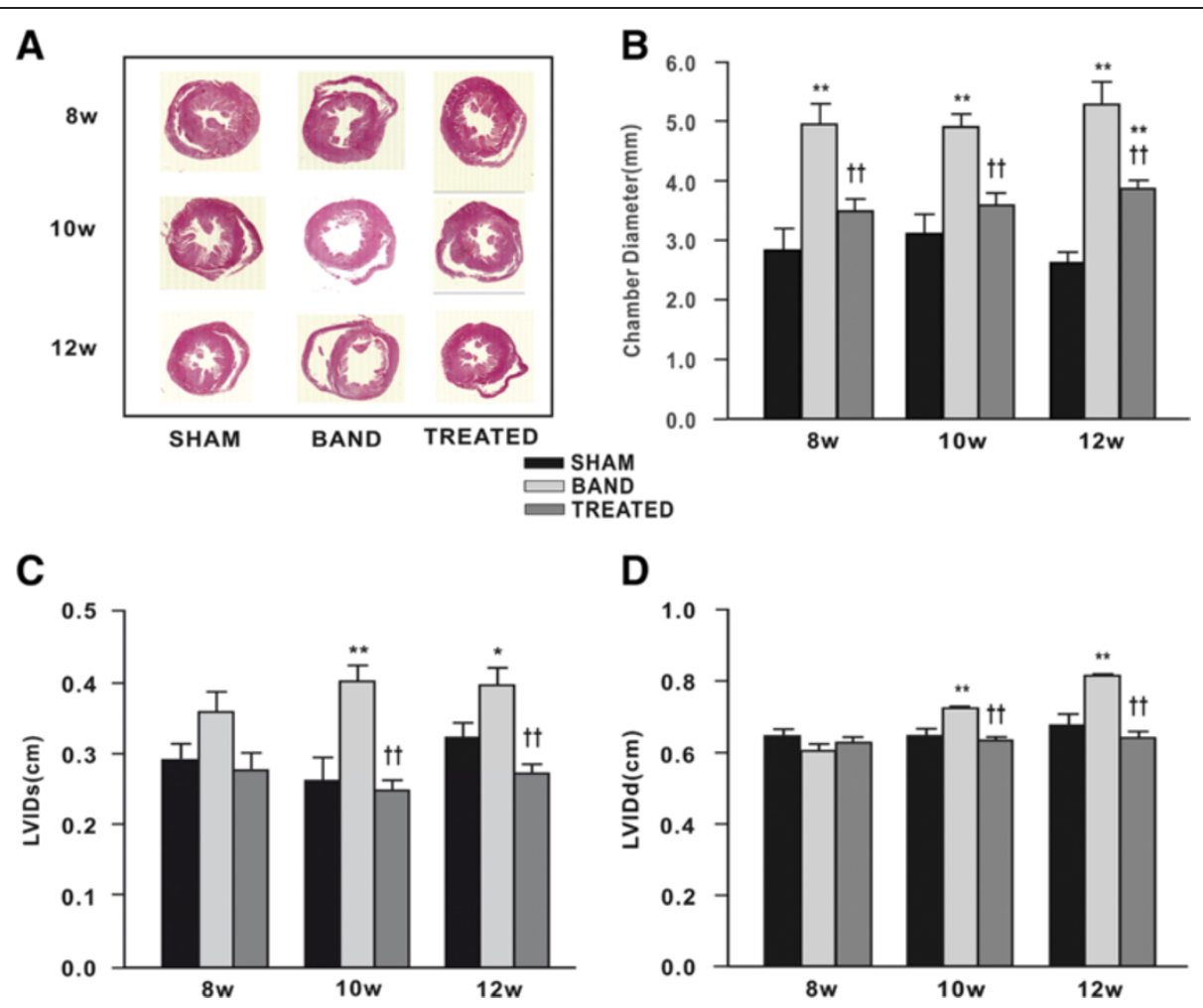

Figure 2 Effect of resveratrol on left ventricular (LV) chamber $(n=4 \sim 7)$. (A) Photomicrographs of the cross sectional area of LV chamber stained by hematoxylin and eosin. (B) Chamber diameter of LV. (C) LV internal dimensions at systole (LVIDs). (D) LV internal dimensions at diastole (LVIDd). Both LVIDs and LVIDd were detected by echocardiographic analysis. Data are mean \pm SEM. ${ }^{*} P<0.05{ }^{* *} P<0.01$ vs. sham rats; ${ }^{\dagger} P<0.05^{\dagger \dagger} P<0.01$ vs. banded rats.

\section{Effect of resveratrol on cardiac fibrosis}

Fibrosis in the cross-sections of heart was stained blue with the Masson's trichrome kit. We detected that both interstitial and perivascular fibrosis were increased in aortic banded rats at 8, 10 and 12 weeks postsurgery respectively, compared to sham rats. Interestingly, resveratrol treatment significantly reduced interstitial fibrosis by $38 \%, 45 \%, 56 \%$ and perivascular fibrosis by $56 \%, 53 \%$, $60 \%$ at 8,10 and 12 weeks postsurgery respectively, compared with banded rats, suggesting that resveratrol treatment can inhibit myocardial fibrosis caused by aortic banding (Figure 4).

\section{Effect of resveratrol on myocardium ultrastructure}

TEM revealed the disrupted myocardium and disorganized mitochondria with abnormal cristae structure in banded rats at 12 weeks postsurgery (Figure 5). Cardiac muscle fibers from sham rats exhibited normal ultrastructural morphology, characterized by laterally aligned myofibrils with highly organized sarcomeres and elongated mitochondria packed tightly in strands running between myofibrils. However, in myocardium from banded rats, myofibrils were fragmented, disrupted and degraded, with disorganized arrays of sarcomeres. Mitochondria appeared rounded or irregular, extensive swelling, abnormally clumped or dispersed, and occasionally broken cristae. Some mitochondria were markedly degraded in cristae with lucent matrix and vacuolation. In myocardium from resveratrol-treated rats, the ultrastructure of cardiomyocytes was effectively maintained and nearly similar to those in sham rats, exhibiting slightly disrupted myofibrils. The mitochondria from resveratrol-treated rats appeared to be irregular shapes, obvious swollen and mildly clumped, but degraded cristae and vacuolations as shown in banded rats were not found in resveratrol-treated rats. Additionally, the apparent loss of myofibrils was only detected in hearts from banded, but not from resveratroltreated rats (Figure 5).

\section{Effect of resveratrol on $\mathrm{Ca}^{2+}$ handling proteins}

The levels of total PLB in hearts from banded rats were markedly increased compared with sham rats, and enhanced by 2.2 -fold especially at 12 weeks postsurgery. Resveratrol treatment continuously alleviated the increased total PLB expression. On the contrary, the levels of p-PLB which were normalized by the levels of GADPH or total PLB were remarkably decreased at 8, 10 and 12 weeks postsurgery in banded rats relative to 

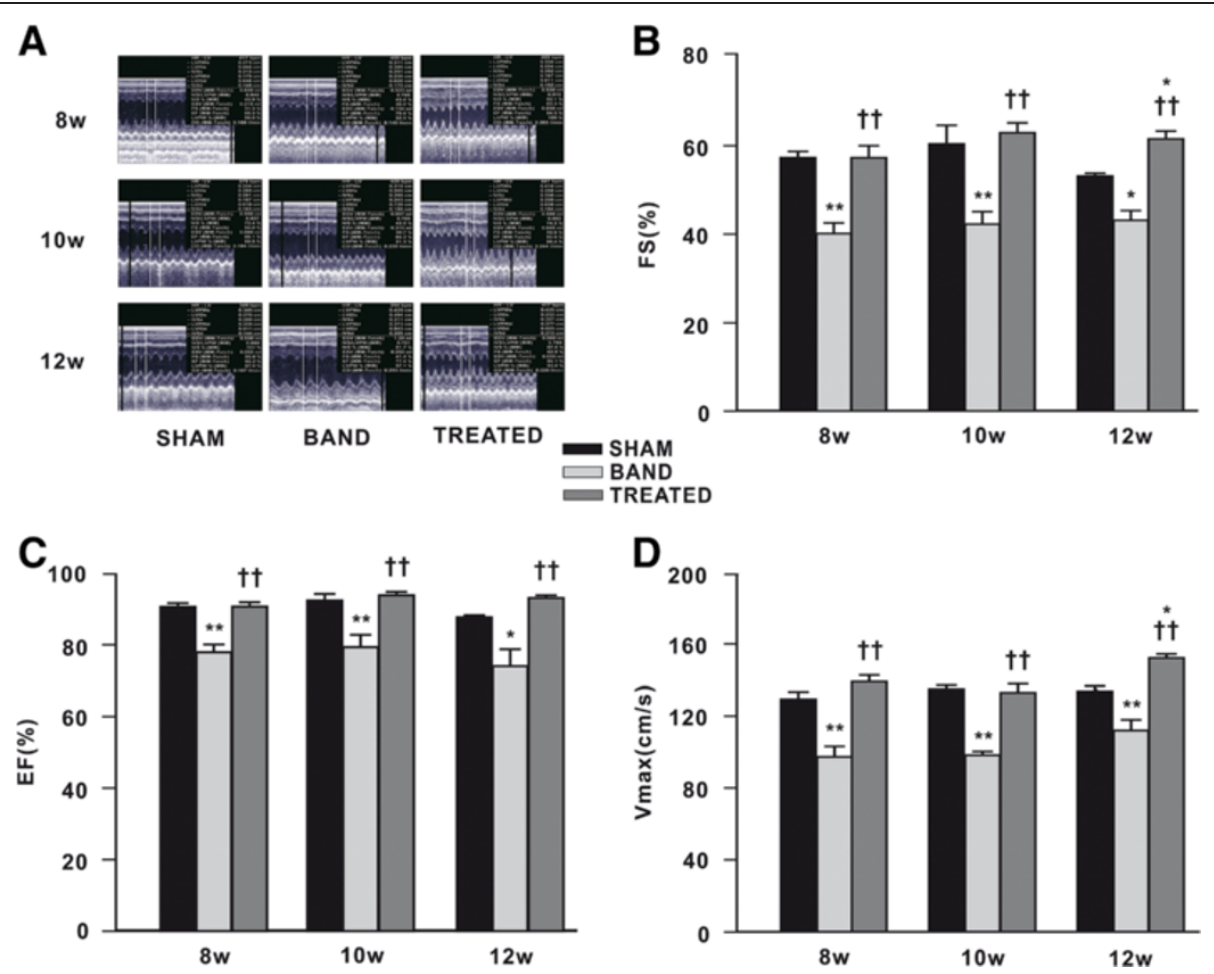

Figure 3 Effect of resveratrol on left ventricular (LV) systolic function ( $n=4 \sim 7)$. (A) Representative pictures of echocardiography. (B) Fractional shortening (FS). (C) Ejection fraction (EF). (D) Maximal velocity through left ventricular outflow tract (Vmax). Data are mean \pm SEM. ${ }^{*} P<0.05{ }^{* *} P<0.01$ vs. sham rats; ${ }^{\dagger} P<0.05{ }^{+\dagger} P<0.01$ vs. banded rats.

sham rats, this effect was prevented by resveratrol treatment (Figure 6A-D).

The levels of $\mathrm{NCX}_{1}$ were increased continuously in banded rats compared with sham rats. Resveratrol completely prevented the upregulation of $\mathrm{NCX}_{1}$ induced by aortic band and even significantly lowered it relative to sham group (Figure 6A, E). On the contrary, SERCA 2 levels were markedly decreased in banded rats compared with sham rats at 8,10 and 12 weeks postsurgery, accompanied with decreased ratio of SERCA $A_{2}$ to PLB and SERCA activity. Notably, resveratrol treatment enhanced the levels of SERCA ${ }_{2}$ and the ratio of SERCA $A_{2}$ to PLB, and prevented the decreased SERCA activity caused by aortic banding (Figure 6A, F-H).

The levels of total CaMKII in hearts from banded rats were unchanged at 8 weeks postsurgery, but increased significantly at 10 and 12 weeks postsurgery compared with sham rats. Resveratrol remarkably alleviated the elevation of total CaMKII induced by aortic banding. Accordingly, the levels of p-CaMKII were progressively increased at 8, 10 and 12 weeks postsurgery in banded rats, which were completely prevented by resveratrol (Figure 7A-C). However, due to sharply rising of total CaMKII, the ratio of p-CaMKII to total CaMKII in banded rats was no different with sham rats at 12 weeks postsurgery, and even lower at 10 weeks postsurgery
(Figure 7A, D). Meanwhile, the significant decreases in $\mathrm{RyR}_{2}$ levels were observed in banded rats compared with sham rats at 8,10 and 12 weeks postsurgery, and the reduction of $\mathrm{RyR}_{2}$ levels induced by aortic banding was prevented by resveratrol treatment (Figure 7A, E).

\section{Discussion}

This study provides several new findings showing that resveratrol prevents the transition from cardiac hypertrophy to HF induced by PO. First, we demonstrated resveratrol reversed LV hypertrophy induced by $\mathrm{PO}$ with time limitation. Second, we showed that resveratrol strongly and continuously prevented the cardiac structural and functional exacerbation due to PO. Third, we showed that resveratrol exerted multiple actions that may contribute to the beneficial effects including (1) inhibition of cardiac fibrosis, (2) prevention of myocardium ultrastructural alterations, (3) modulation of activation and expression of $\mathrm{Ca}^{2+}$ handling proteins.

\section{Antihypertrophic effect of resveratrol has a time limit}

Antihypertrophic effect of resveratrol and its analogue has been documented in different experimental settings of PO [1,3,4,8-12]. In this study, 4 weeks after aortic banding surgery, rats developed PO-induced centric hypertrophy, as indicated by significant increase in wall 

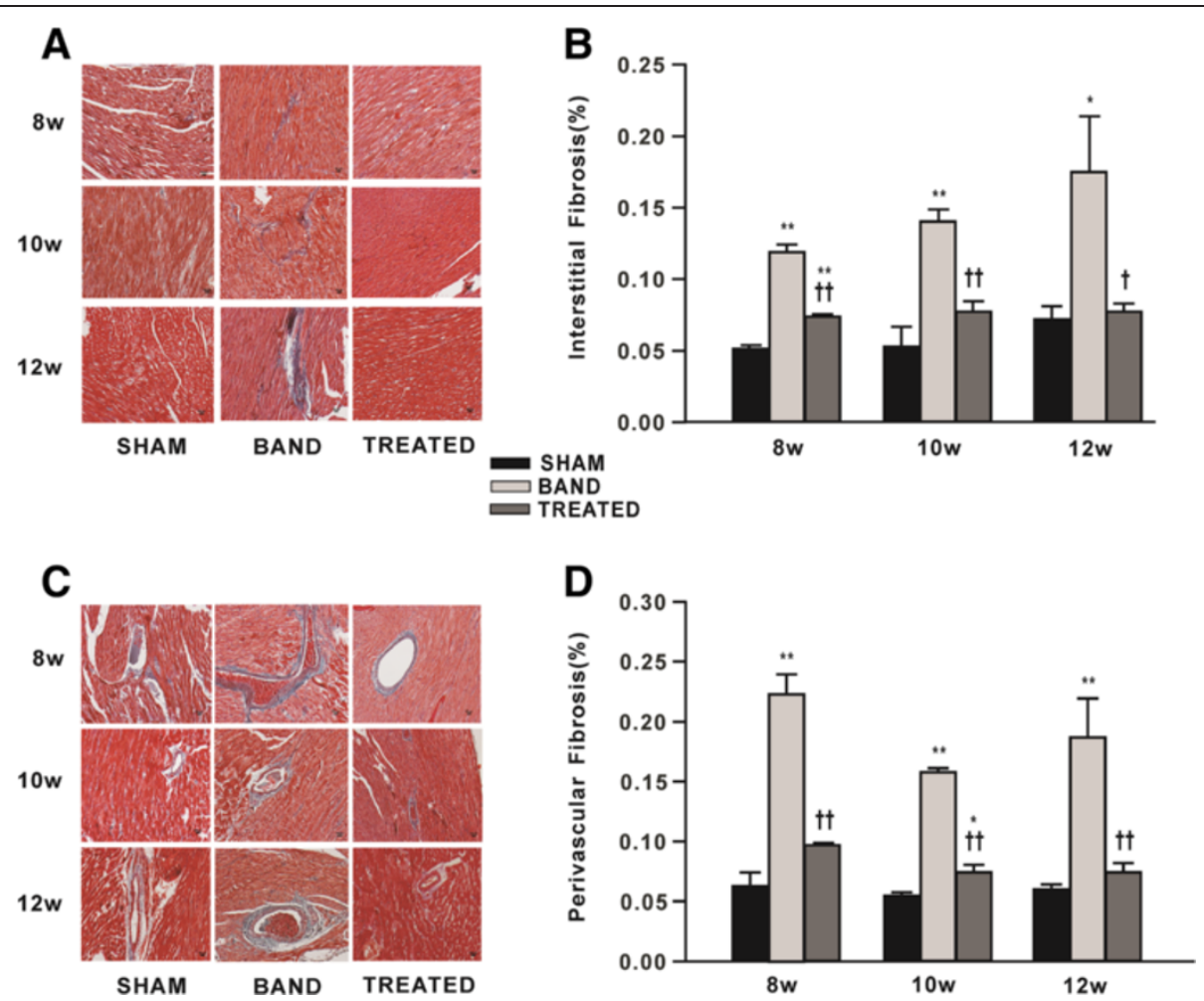

Figure 4 Effect of resveratrol on cardiac fibrosis in left ventricular (LV) myocardium $(n=4)$. (A) Representative photomicrographs of Masson trichrome staining for interstitial fibrosis showing myocardium in red and fibrosis in blue. (B) Quantification of interstitial fibrosis. (C) Representative photomicrographs of Masson trichrome staining for perivascular fibrosis. (D) Quantification of perivascular fibrosis. Data are mean \pm SEM. ${ }^{*} P<0.05,{ }^{* *} P<0.01$ vs. sham rats; ${ }^{\dagger} P<0.05,{ }^{\dagger+} P<0.01$ vs. banded rats.

thickness without chamber dilation. Consistent with previous studies, resveratrol treatment regressed developed cardiac hypertrophy at 8 and 10 weeks postsurgery. However, resveratrol did not prevent cardiac hypertrophy at the late stage of the experiment, as shown by histological analysis. The data of $\mathrm{LVm} / \mathrm{BW}$ also suggested that the effect of resveratrol was receded gradually as PO was sustained. Developed cardiac hypertrophy in treated rats was further confirmed by echocardiography analysis, which showed a marked increase in wall thickness compared with sham rats at 12 weeks postsurgery (see Additional file 2). This novel finding indicated that the timing of treatment is a critical factor for antihypertrophic effect of resveratrol.

\section{Resveratrol prevented the development of decompensatory phase}

4-week aortic banding caused compensatory centric hypertrophy characterized by high contractile function of LV, increased thickness of LV wall and decreased LVIDs. However, with long-standing untreated PO, LV systolic performance decreased and LV dilatation occurred, which are the key features of decompensatory phase, namely HF. Resveratrol treatment effectively preserved cardiac systolic function and LV chamber dimension. Previous studies demonstrated that resveratrol improved contractile function with abolishing cardiac hypertrophy caused by PO [1,3]. Interestingly, we found that resveratrol continuously exerted cardioprotective effects in banded rats and these effects sustained to decompensatory stage of HF even though its antihypertrophic effect was absent. Similarly, Ste'phanie Rimbaud found that resveratrol treatment exerted beneficial protective effects on survival and cardiac contraction in DSS rats fed with HS-NT which is a hypertensive model of HF [5]. However, the mechanism underlying the development of HF in DSS rats is apparently different from aortic banded rats.

\section{Cardioprotective mechanisms of resveratrol}

The development of HF is associated with marked myocardial fibrosis which is characterized by excessive extracellular matrix (ECM) deposition and myocardial stiffness [13]. Limiting pathological myocardial fibrosis represents a potential therapeutic target to prevent HF. Previous studies have demonstrated that resveratrol has beneficial effects to reduce cardiac fibrosis in a variety of pathological models $[14,15]$. For instance, resveratrol alleviated cardiac fibrosis in spontaneously hypertensive rats [16] and decreased left ventricular interstitial and perivascular fibrosis 


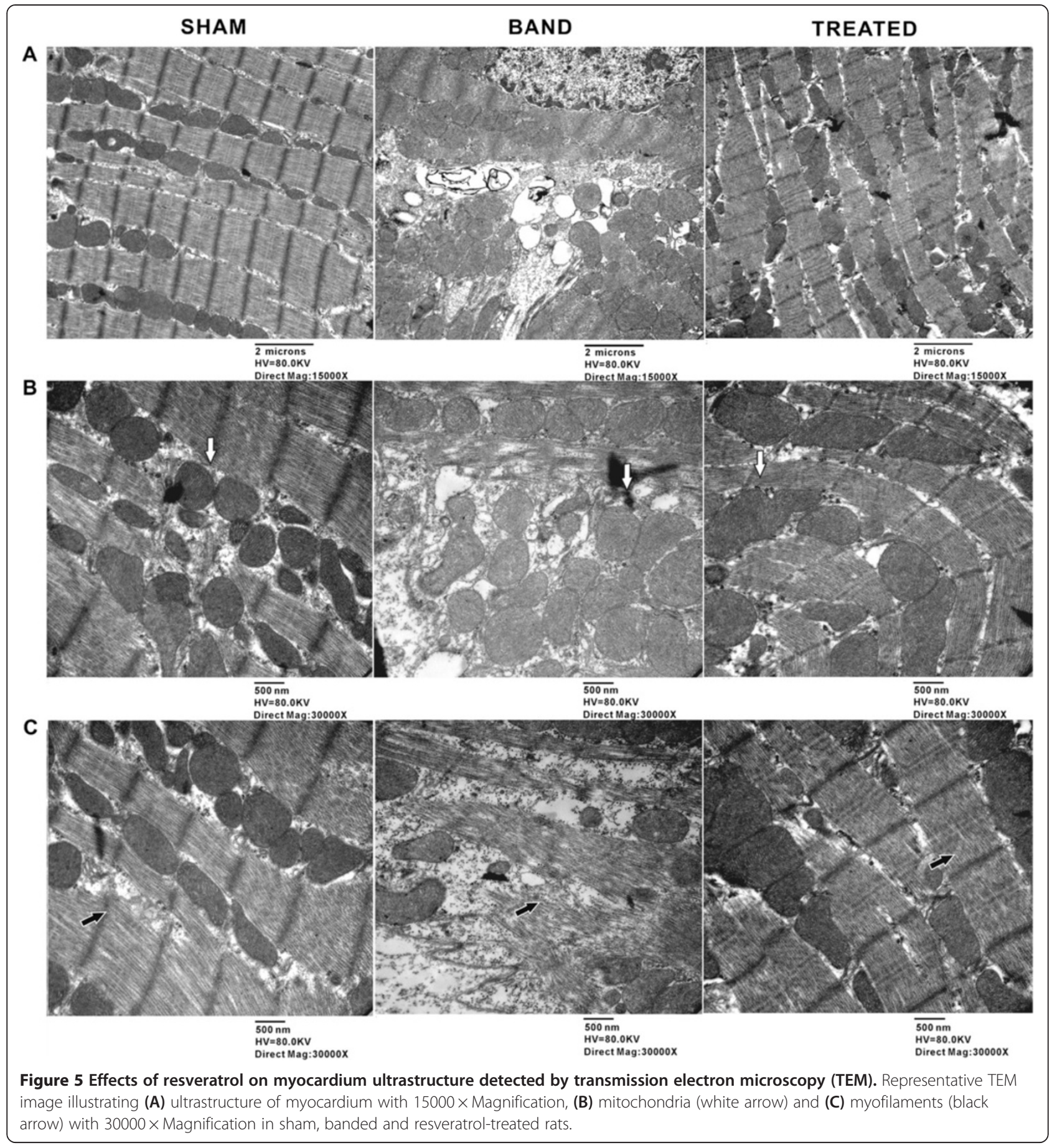

in DOCA-salt rats [17]. We demonstrated that resveratrol inhibited interstitial and perivascular fibrosis due to PO, indicative of the improvement of myocardial compliance and diastolic function. Indeed, increased LV pressure halftime, an index of diastolic heart function, was detected in banded rats, but prevented by resveratrol (see Additional file 3). Meanwhile, inhibition of cardiac fibrosis by resveratrol could explain the findings that $\mathrm{LVm} / \mathrm{BW}$ in treated rats was remarkably lower than in banded rats at 12 weeks postsurgery while no difference in the size of cardiomyocytes was detected by histological analysis between two groups.

Second, we found that resveratrol protected against myocardial ultrastructural abnormalities induced by PO. The most obvious detriment to myocardial ultrastructure induced by PO was decrease of myofibrils as a result of rupture and degradation of myofilaments. Notably, resveratrol treatment normalized myocardial ultrastructure as 

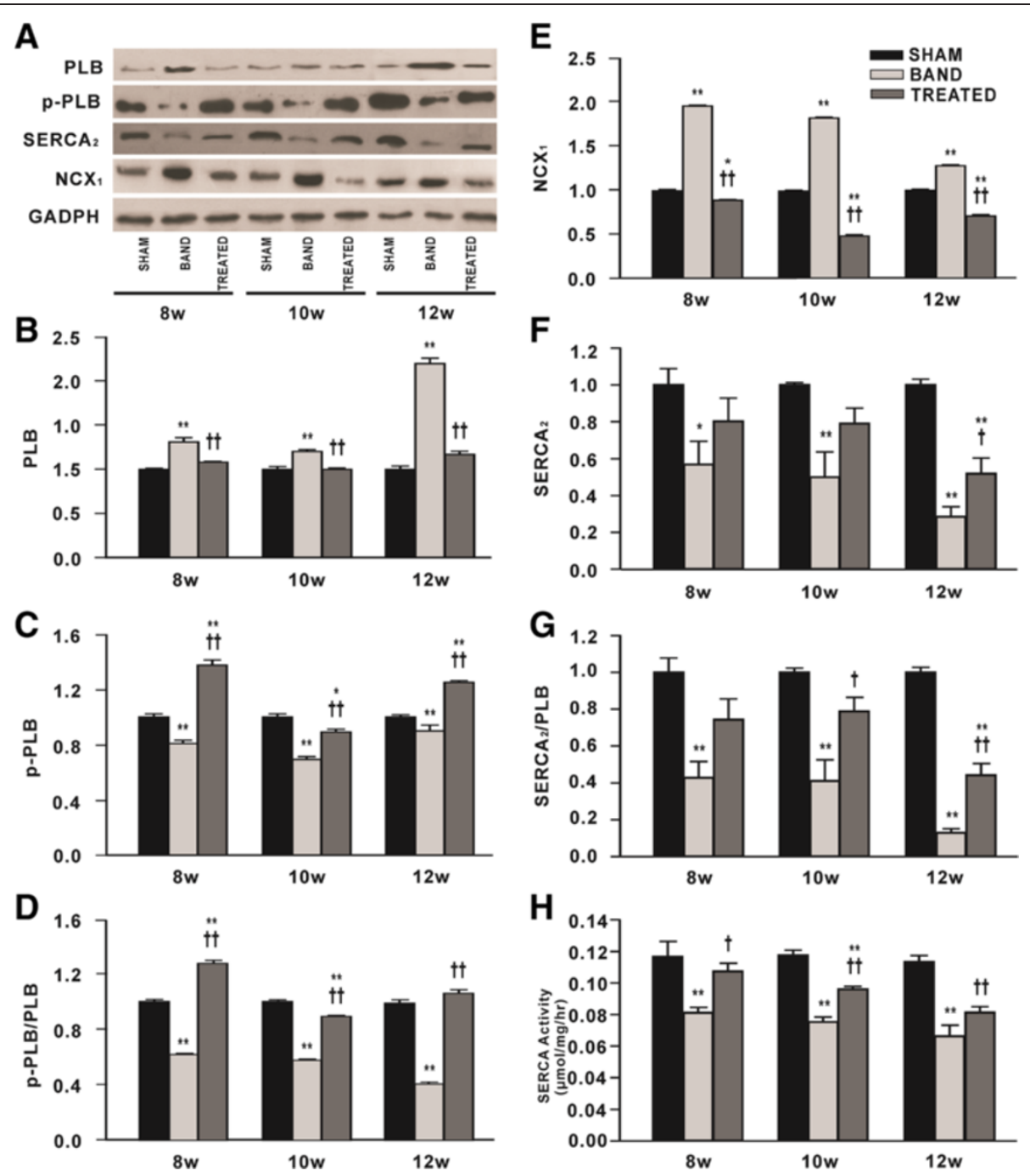

Figure 6 Effects of resveratrol on the expressions of proteins responsible for $\mathrm{SR} \mathrm{Ca}^{2+}$ load and sarcoplasmic reticulum Ca ${ }^{2+}$-ATPase activity $(\mathbf{n}=\mathbf{3})$. (A) Representative western blot from each group. (B) The levels of Phospholamban (PLB). (C) The levels of Phospho-PLB (S16) (p-PLB). (D) The ratio of p-PLB to PLB. (E) The levels of $\mathrm{Na}^{+} / \mathrm{Ca}^{2+}$-exchangers $\left(\mathrm{NCX}_{1}\right.$ ). (F) The levels of Sarcoplasmic reticulum Ca ${ }^{2+}$-ATPase (SERCA 2 ). (G) The ratio of SERCA 2 to PLB. (H) SERCA activity. Levels of proteins were quantified by densitometry and normalized against GADPH. Data are mean \pm SEM. ${ }^{*} P<0.05^{* *} P<0.01$ vs. sham rats; ${ }^{\dagger} P<0.05^{{ }^{+\dagger}} P<0.01$ vs. banded rats.

evidenced by intact myofibrils and organized sarcomeres, which may be attributed to multiple mechanisms beneficial to cell survival including prevention of cardiomyocyte apoptosis, regulation of autophagy, and reduction of oxidative stress [14,18-20]. The other striking abnormality caused by PO was swollen and disordered mitochondria revealed by TEM. Resveratrol only partially prevented mitochondrial lesions. However, other studies demonstrated that resveratrol significantly attenuated the abnormality of mitochondrial ultrastructure in sepsis-induced myocardial depression with a large dose $(30 \mathrm{mg} / \mathrm{kg}$ or $60 \mathrm{mg} / \mathrm{kg}$ ) [21], and preserved mitochondrial function and biogenesis contributing to improve cardiac energy metabolism and reduce oxidative stress in other models $[5,20,22,23]$. This difference could be explained by the diversity of resveratrol dosage, the period of treatment, the stage of HF and animal models.

$\mathrm{Ca}^{2+}$ cycling which refers to the release and reuptake of intracellular $\mathrm{Ca}^{2+}$ is highly regulated in cardiomyocytes and determines the process of cardiac muscle contraction and relaxation. Defects in the regulation of $\mathrm{Ca}^{2+}$ handling proteins contribute to HF. To our knowledge, the effects of resveratrol on $\mathrm{Ca}^{2+}$ handling proteins in $\mathrm{HF}$ remain unclear. Impaired $\mathrm{SERCA}_{2}$ function and enhanced NCX activity have been proposed as causes of reduced $\mathrm{SR} \mathrm{Ca}^{2+}$ load in HF. Moreover, PLB, a regulator of the affinity of $\mathrm{SERCA}_{2}$ for $\mathrm{Ca}^{2+}$, balances SR $\mathrm{Ca}^{2+}$ uptake through inhibiting the affinity of $\mathrm{SERCA}_{2}$ for $\mathrm{Ca}^{2+}$ by unphosphorylated PLB and relieving this inhibition by phosphorylation. As a negative regulator of SERCA $A_{2}$, hypophosphorylated 

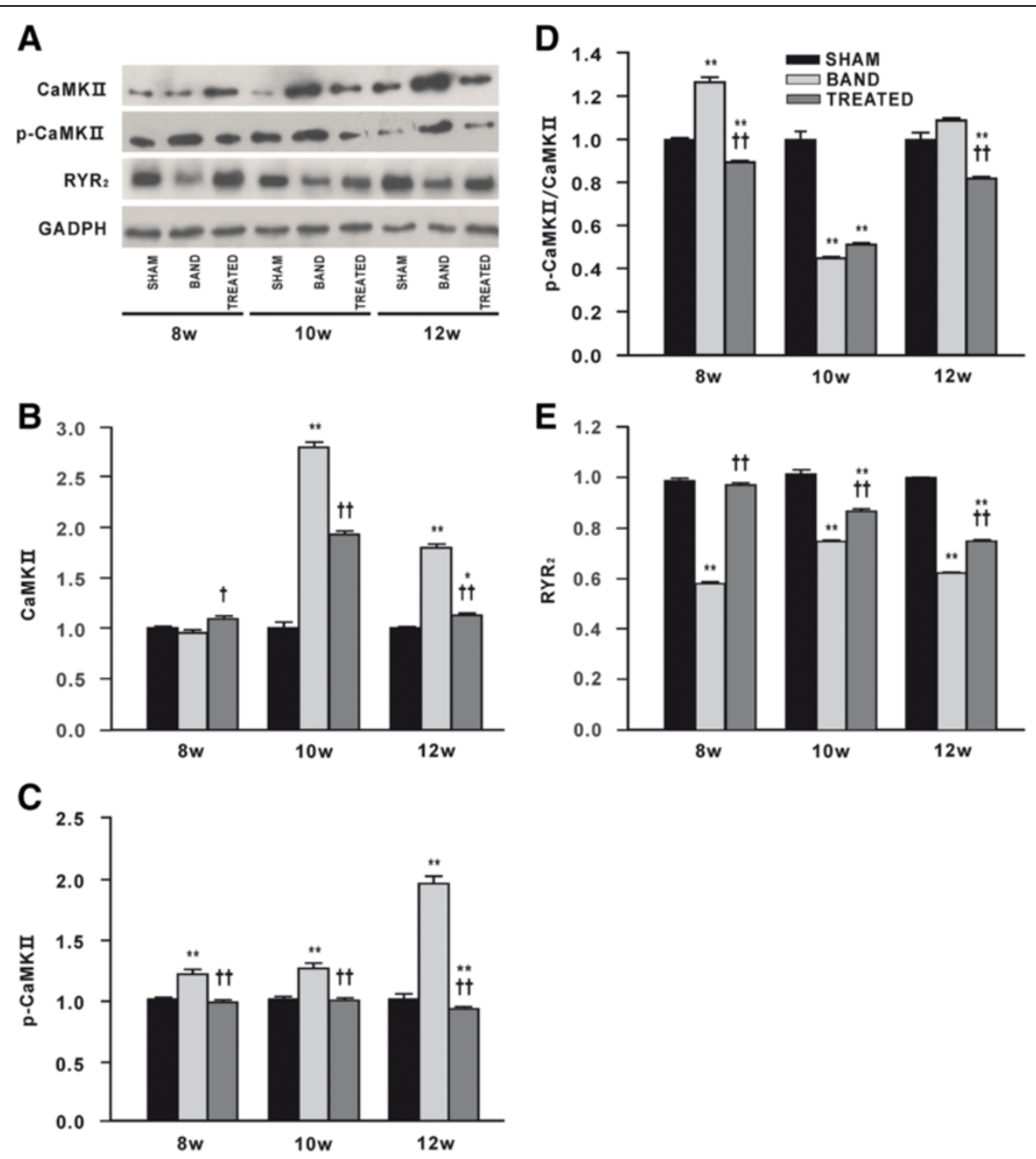

Figure 7 Effects of resveratrol on protein expressions of $\mathrm{Ca}^{2+} /$ calmodulin-dependent protein kinase II (CaMK II), phospho-CaMKII (Thr286) (p-CaMK II) and ryanodine receptor $\left(\operatorname{RyR}_{2}\right)(\mathbf{n}=3)$. (A) Representative western blot from each group. (B) The levels of CaMK II. (C) The levels of p-CaMK II. (D) The ratio of p-CaMK II to CaMK II. (E) The levels of RyR2. Levels of proteins were quantified by densitometry and normalized against GADPH. Data are mean \pm SEM. ${ }^{*} P<0.05{ }^{* *} P<0.01$ vs. sham rats; ${ }^{\dagger} P<0.05{ }^{+\dagger} P<0.01$ vs. banded rats.

PLB is an important cause of deficient SR $\mathrm{Ca}^{2+}$ uptake in failing hearts. Accordingly, both SERCA 2 overexpression and PLB inhibition have been designed as therapeutic strategies for HF [6]. In this study, PO caused substantially decreased SERCA $\mathrm{A}_{2}$ expression and ATPase activity, elevated $\mathrm{NCX}_{1}$, increased PLB and decreased $\mathrm{p}-\mathrm{PLB}$, indicating that $\mathrm{SR} \mathrm{Ca}^{2+}$ load in this animal model is deficient, consequently leading to impaired contractile function. Resveratrol effectively upregulated SERCA ${ }_{2}$ levels, ATPase activity and the ratio of phosphorylated to unphosphorylated PLB, accompanied by a significant downregulation of $\mathrm{NCX}_{1}$, indicative of improved $\mathrm{SR} \mathrm{Ca}^{2+}$ load in hearts. Consistent with our results, a previous study showed that increased $\mathrm{SERCA}_{2 \mathrm{a}}$ expression by resveratrol improved contractile function in chronic type 1 diabetes [12].
Additionally, we detected a significant increase in expression and phosphorylation of CaMKII due to PO. It has been reported that CaMKII hyperphosphorylation of $\mathrm{RyR}_{2}$ accounts for excessive diastolic SR $\mathrm{Ca}^{2+}$ leak in non-ischemic (aortic banding) cardiomyopathy, but not in ischemia (post-MI) in which PKA phosphorylation of $\mathrm{RyR}_{2}$ is involved [24]. This leads to increased $\mathrm{RyR}_{2}$ open probability and a diastolic SR $\mathrm{Ca}^{2+}$ leak because of a higher sensitivity to $\mathrm{Ca}^{2+}$-induced $\mathrm{Ca}^{2+}$ release at low cytoplasmic $\mathrm{Ca}^{2+}$ concentrations. In our experiment, resveratrol attenuated the increase in CaMKII level and completely inhibited hyperphosphorylation of CaMKII. It has been demonstrated that $\mathrm{RyR}_{2}$ leak was inhibited through inhibition of CaMKII phosphorylation. Knockin mice with an inactivated CaMKII phosphorylation site 
on $\mathrm{RyR}_{2}$ had lower $\mathrm{SR} \mathrm{Ca}^{2+}$ leak and improved SR $\mathrm{Ca}^{2+}$ load, and were relatively protected from HF development after transverse aortic constriction [25]. In patients with HF, CaMKII- but not PKA-dependent RyR $\mathrm{R}_{2}$ phosphorylation was significantly increased, accompanied by increased $\mathrm{SR} \mathrm{Ca}^{2+}$ leak, reduced systolic $\mathrm{Ca}^{2+}$ transients, depletion of $\mathrm{SR} \mathrm{Ca}^{2+}$ storage and elevated diastolic $\mathrm{Ca}^{2+}$ levels. Moreover, CaMKII inhibition, but not inhibition of PKA yielded a reduction of the SR $\mathrm{Ca}^{2+}$ leak [25]. Therefore, we speculate that CaMKII downregulation by resveratrol plays a major role in preventing the development of cardiac dysfunction in aortic banded animals via inhibition of $\mathrm{RyR}_{2}$ leak.

Although the activity of $\mathrm{RyR}_{2}$ channel in HF was investigated in many studies, very little is known about expression of $\mathrm{RyR}_{2}$ and its significance in failing heart. We found that $\mathrm{RyR}_{2}$ protein expression was significantly decreased in myocardium of HF and resveratrol effectively enhanced the expression of $\mathrm{RyR}_{2}$. We speculate that downregulation of $\mathrm{RyR}_{2}$ may decrease systolic $\mathrm{Ca}^{2+}$ release and impair cardiac contractility. Similarly, Kubalova et al. found that $\mathrm{RyR}_{2}$ content in failing hearts was decreased to approximately half of control values, but the levels of other proteins of the $\mathrm{Ca}^{2+}$ release channel complex such as triadin and junctin were not changed. The altered stoichiometry of triadin and junctin to $\mathrm{RyR}_{2}$ may increase activity of $\mathrm{RyR}_{2}$, thus leading to the abnormal $\mathrm{Ca}^{2+}$ handling in HF [26].

A prevailing theory is that one of the major mechanisms proposed to underlie resveratrol mediated cardioprotection is reduction of oxidative stress $[4,27,28]$. Polydatin, a resveratrol glucoside, has been shown to prevent enhanced $\mathrm{Ca}^{2+}$ spark-mediated SR leak by reducing oxidative stress in $\mathrm{RyR}_{2}$ in burn-traumatized heart, leading to protection of cardiac function against burn injury [29]. Further studies are needed to understand whether reduction of oxidative stress by resveratrol could regulate $\mathrm{RyR}_{2}$ and other $\mathrm{Ca}^{2+}$ handling proteins in aortic banded rats.

\section{Conclusions}

These data demonstrated that resveratrol treatment prevented cardiac hypertrophy induced by PO with timelimitation and inhibited the development of HF via improving cardiac structure and function. The novel findings on mechanisms underlying cardioprotection of resveratrol include protection of myocardium ultrastructure and regulation of global $\mathrm{Ca}^{2+}$ handling proteins.

\section{Additional files}

Additional file 1: Two-dimensionally guided M-mode echocardiographic measurement of wall thickness (IVSs, IVSd, LVPWs and LVPWd) (A and B), chamber dimensions (LVIDs and
LVIDd) (A and C), systolic parameters (FS and EF) (A and D) of left ventricular (LV) in sham and banded rats at 4 weeks postsurgery ( $\mathbf{n}=6$ 13). Data are mean \pm SEM. ${ }^{*} P<0.05{ }^{* *} P<0.01$ vs. sham rats.

Additional file 2: Two-dimensionally guided M-mode echocardiographic measurement of IVSd (A), IVSs (B), LVPWd (C) and LVPWs (D) in sham, banded and resveratrol-treated rats at 8,10 and 12 weeks postsurgery $(n=4 \sim 7)$. Data are mean \pm SEM. ${ }^{*} P<0.05$ vs. sham rats; ${ }^{+} P<0.05$ vs. banded rats.

Additional file 3: Effect of resveratrol on left ventricular (LV) cardiac output and pressure half-time $(n=4 \sim 7)$. (A) Representative pictures of echocardiography. (B) Cardiac output. (C) Pressure half-time. Data are mean \pm SEM. ${ }^{*} P<0.05^{* *} P<0.01$ vs. sham rats; ${ }^{\dagger} P<0.05{ }^{\dagger+} P<0.01$ vs. banded rats.

\section{Abbreviations}

HF: Heart failure; PO: Pressure overload; SR: Sarcoplasmic reticulum; LV: Left ventricular; FS: Fractional shortening; EF: Ejection fraction; Vmax: Maximal velocity through left ventricular outflow tract; LVIDd and LVIDs: LV internal dimensions at both diastole and systole; LVPWd and LVPWs: LV posterior wall dimensions at both diastole and systole; IVSd and IVSs: Interventricular septal dimensions at both diastole and systole; LVm/BW: LV mass-to-body weight ratio; TEM: Transmission electron microscopy; CaMKII: $\mathrm{Ca}^{2+} / \mathrm{calmodulin}$ dependent protein kinase II; p-CaMKII: Phospho-CaMKII (Thr286);

SERCA $A_{2}$ : Sarcoplasmic reticulum Ca ${ }^{2+}$ ATPase 2 ; RyR: Ryanodine receptor ${ }_{2 i}$; PLB: Phospholamban; $\mathrm{NCX}_{1}: \mathrm{Na}^{+} / \mathrm{Ca}^{2+}$-exchangers; p-PLB: Phospho-PLB (S16).

Competing interests

The authors declare that they have no competing interests.

\section{Authors' contributions}

$\mathrm{QD}, \mathrm{ZW}, \mathrm{XL}$ and $\mathrm{MC}$ designed the research project; ZW performed all experimental tests. LZ provided essential reagents and essential materials; JL and JD assisted in surgeries and echocardiography; QD and MC analyzed all data; QD performed all statistical analyses; QD and MC wrote the manuscript and have primary responsibility for final content; QD and ZW produced all figures. JY and CY assisted in manuscript writing and figure producing; all authors read and approved the final manuscript.

\section{Acknowledgements}

This project was supported by National Natural Science Foundation in China (30440053 and 30570759, grants to M. C.), Natural Science Foundation of Guang Dong Province in China (\$2011010004269, grants to M. C.) and Technology Planning Project of Guangdong Province in China (2013B022000099, grants to X. L.).

\section{Author details}

'Department of Physiology, Guangzhou Medical University, Guangzhou 510182, China. ²Guangzhou Institute of Cardiovascular Disease, Guangzhou 510260, China. ${ }^{3}$ Southern Medical University, 1838 Guang zhou da dao bei, Guangzhou 510515, China. ${ }^{4}$ Department of Cardiology, The Second Hospital Affiliated with Guangzhou Medical University, Guangzhou 510260, China. ${ }^{5}$ Experimental Medical Research Center, Guangzhou Medical University, Guangzhou 510182, China. ${ }^{6}$ Department of Neurology, The Fifth Hospital Affiliated with Zunyi Medical College, Zhuhai 519100, China.

Received: 4 September 2014 Accepted: 10 November 2014

Published online: 26 November 2014

\section{References}

1. Wojciechowski P, Juric D, Louis XL, Thandapilly SJ, Yu L, Taylor C, Netticadan $T$ : Resveratrol arrests and regresses the development of pressure overload- but not volume overload-induced cardiac hypertrophy in rats. J Nutr 2010, 140:962-968.

2. Thandapilly SJ, LeMaistre JL, Louis XL, Anderson CM, Netticadan T, Anderson HD: Vascular and cardiac effects of grape powder in the spontaneously hypertensive rat. Am J Hypertens 2012, 25:1070-1076.

3. Juric D, Wojciechowski P, Das DK, Netticadan T: Prevention of concentric hypertrophy and diastolic impairment in aortic-banded rats treated with resveratrol. Am J Physiol Heart Circ Physiol 2007, 292:H2138-H2143. 
4. Thandapilly SJ, Wojciechowski P, Behbahani J, Louis XL, Yu L, Juric D, Kopilas MA, Anderson HD, Netticadan T: Resveratrol prevents the development of pathological cardiac hypertrophy and contractile dysfunction in the SHR without lowering blood pressure. Am J Hypertens 2010, 23:192-196.

5. Rimbaud S, Ruiz M, Piquereau J, Mateo P, Fortin D, Veksler V, Garnier A, Ventura-Clapier R: Resveratrol improves survival, hemodynamics and energetics in a rat model of hypertension leading to heart failure. PLOS One 2011, 6:e26391.

6. Marks AR: Calcium cycling proteins and heart failure: mechanisms and therapeutics. J Clin Invest 2013, 123:46-52.

7. Lizotte $E$, Tremblay A, Allen BG, Fiset C: Isolation and characterization of subcellular protein fractions from mouse heart. Anal Biochem 2005, 345:47-54

8. Thandapilly SJ, Louis XL, Yang T, Stringer DM, Yu L, Zhang S, Wigle J, Kardami E, Zahradka P, Taylor C, Anderson HD, Netticadan T: Resveratrol prevents norepinephrine induced hypertrophy in adult rat cardiomyocytes, by activating NO-AMPK pathway. Eur J Pharmacol 2011, 668:217-224.

9. Opie LH, Lecour S: The red wine hypothesis: from concepts to protective signalling molecules. Eur Heart J 2007, 28:1683-1693.

10. Dolinsky WW, Chan AY, Robillard Frayne I, Light PE, Des Rosiers C, Dyck JR: Resveratrol prevents the prohypertrophic effects of oxidative stress on LKB1. Circulation 2009, 119:1643-1652.

11. Ding DF, You N, Wu XM, Xu JR, Hu AP, Ye XL, Zhu Q, Jiang XQ, Miao H, Liu C, Lu YB: Resveratrol attenuates renal hypertrophy in early-stage diabetes by activating AMPK. Am J Nephrol 2010, 31:363-374.

12. Sulaiman M, Matta MJ, Sunderesan NR, Gupta MP, Periasamy M, Gupta M: Resveratrol, an activator of SIRT1, upregulates sarcoplasmic calcium ATPase and improves cardiac function in diabetic cardiomyopathy. Am J Physiol Heart Circ Physiol 2010, 298:H833-H843.

13. Conrad CH, Brooks WW, Hayes JA, Sen S, Robinson KG, Bing OH: Myocardial fibrosis and stiffness with hypertrophy and heart failure in the spontaneously hypertensive rat. Circulation 1995, 91:161-170.

14. Tanno M, Kuno A, Yano T, Miura T, Hisahara S, Ishikawa S, Shimamoto K, Horio $Y$ : Induction of manganese superoxide dismutase by nuclear translocation and activation of SIRT1 promotes cell survival in chronic heart failure. J Biol Chem 2010, 285:8375-8382.

15. Qin F, Siwik DA, Luptak I, Hou X, Wang L, Higuchi A, Weisbrod RM, Ouchi N, Tu VH, Calamaras TD, Miller EJ, Verbeuren TJ, Walsh K, Cohen RA, Colucci WS: The polyphenols resveratrol and S17834 prevent the structural and functional sequelae of diet-induced metabolic heart disease in mice. Circulation 2012, 125:1757-1764. S1-6.

16. Thandapilly SJ, Louis XL, Behbahani J, Movahed A, Yu L, Fandrich R, Zhang S, Kardami E, Anderson HD, Netticadan T: Reduced hemodynamic load aids low-dose resveratrol in reversing cardiovascular defects in hypertensive rats. Hypertens Res 2013, 36:866-872.

17. Chan V, Fenning A, lyer A, Hoey A, Brown L: Resveratrol improves cardiovascular function in DOCA-salt hypertensive rats. Curr Pharm Biotechnol 2011, 12:429-436.

18. Mukherjee S, Dudley J, Das DK: Dose-dependency of resveratrol in providing health benefits. Dose Response 2010, 8:478-500.

19. Sundaresan NR, Pillai VB, Gupta M: Emerging roles of SIRT1 deacetylase in regulating cardiomyocyte survival and hypertrophy. J Mol Cell Cardiol 2011, 51:614-618.

20. Danz ED, Skramsted J, Henry N, Bennett JA, Keller RS: Resveratrol prevents doxorubicin cardiotoxicity through mitochondrial stabilization and the Sirt1 pathway. Free Radic Biol Med 2009, 46:1589-1597.

21. Smeding L, Leong-Poi H, Hu P, Shan Y, Haitsma JJ, Horvath E, Furmli S, Masoom H, Kuiper JW, Slutsky AS, Parker TG, Plotz FB, dos Santos CC: Salutary effect of resveratrol on sepsis-induced myocardial depression. Crit Care Med 2012, 40:1896-1907.

22. Sin TK, Yu AP, Yung BY, Yip SP, Chan LW, Wong CS, Ying M, Rudd JA, Siu PM: Modulating effect of SIRT1 activation induced by resveratrol on Foxo1-associated apoptotic signalling in senescent heart. J Physiol 2014, 592:2535-2548.

23. Shinmura K, Tamaki K, Sano M, Nakashima-Kamimura N, Wolf AM, Amo T, Ohta S, Katsumata Y, Fukuda K, Ishiwata K, Suematsu M, Adachi T: Caloric restriction primes mitochondria for ischemic stress by deacetylating specific mitochondrial proteins of the electron transport chain. Circ Res 2011, 109:396-406.

24. Respress JL, van Oort RJ, Li N, Rolim N, Dixit SS, deAlmeida A, Voigt N, Lawrence WS, Skapura DG, Skardal K, Wisloff U, Wieland T, Ai X, Pogwizd
SM, Dobrev D, Wehrens XH: Role of RyR2 phosphorylation at S2814 during heart failure progression. Circ Res 2012, 110:1474-1483.

25. Fischer TH, Herting J, Tirilomis T, Renner A, Neef S, Toischer K, Ellenberger D, Forster A, Schmitto JD, Gummert J, Schondube FA, Hasenfuss G, Maier LS, Sossalla S: Ca2+/calmodulin-dependent protein kinase II and protein kinase A differentially regulate sarcoplasmic reticulum $\mathrm{Ca} 2+$ leak in human cardiac pathology. Circulation 2013, 128:970-981.

26. Kubalova Z, Terentyev D, Viatchenko-Karpinski S, Nishijima Y, Gyorke I, Terentyeva R, da Cunha DN, Sridhar A, Feldman DS, Hamlin RL, Carnes CA, Gyorke S: Abnormal intrastore calcium signaling in chronic heart failure. Proc Natl Acad Sci U S A 2005, 102:14104-14109.

27. Shigematsu S, Ishida S, Hara M, Takahashi N, Yoshimatsu H, Sakata T, Korthuis RJ: Resveratrol, a red wine constituent polyphenol, prevents superoxidedependent inflammatory responses induced by ischemia/reperfusion, platelet-activating factor, or oxidants. Free Radic Biol Med 2003, 34:810-817.

28. Tatlidede E, Sehirli O, Velioglu-Ogunc A, Cetinel S, Yegen BC, Yarat A, Suleymanoglu S, Sener G: Resveratrol treatment protects against doxorubicin-induced cardiotoxicity by alleviating oxidative damage. Free Radic Res 2009, 43:195-205.

29. Jiang X, Liu W, Deng J, Lan L, Xue X, Zhang C, Cai G, Luo X, Liu J: Polydatin protects cardiac function against burn injury by inhibiting sarcoplasmic reticulum $\mathrm{Ca} 2+$ leak by reducing oxidative modification of ryanodine receptors. Free Radic Biol Med 2013, 60:292-299.

doi:10.1186/s12967-014-0323-x

Cite this article as: Dong et al:: Resveratrol ameliorates cardiac dysfunction induced by pressure overload in rats via structural protection and modulation of $\mathrm{Ca}^{2+}$ cycling proteins. Journal of Translational Medicine 2014 12:323.

\section{Submit your next manuscript to BioMed Central and take full advantage of:}

- Convenient online submission

- Thorough peer review

- No space constraints or color figure charges

- Immediate publication on acceptance

- Inclusion in PubMed, CAS, Scopus and Google Scholar

- Research which is freely available for redistribution 